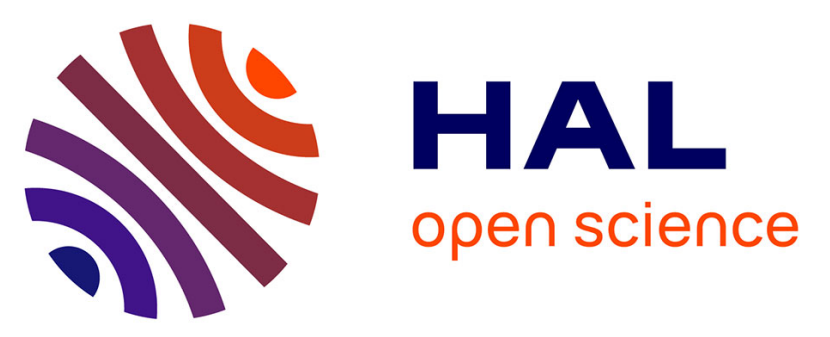

\title{
Interplay between bandwidth-controlled and filling-controlled pressure-induced Mott insulator to metal transition in the molecular compound [Au(Et-thiazdt)_2]
}

Benjamin Brière, Jonathan Caillaux, Yann Le Gal, Dominique Lorcy, S. Lupi, A. Perucchi, Mustapha Zaghrioui, Jean-Claude Soret, Rodolphe Sopracase, Vinh Ta-Phuoc

\section{- To cite this version:}

Benjamin Brière, Jonathan Caillaux, Yann Le Gal, Dominique Lorcy, S. Lupi, et al.. Interplay between bandwidth-controlled and filling-controlled pressure-induced Mott insulator to metal transition in the molecular compound [Au(Et-thiazdt)_2]. Physical Review B, 2018, 97 (3), pp.035101. 10.1103/PhysRevB.97.035101 . hal-01685695

\section{HAL Id: hal-01685695}

https://hal-univ-rennes1.archives-ouvertes.fr/hal-01685695

Submitted on 29 May 2018

HAL is a multi-disciplinary open access archive for the deposit and dissemination of scientific research documents, whether they are published or not. The documents may come from teaching and research institutions in France or abroad, or from public or private research centers.
L'archive ouverte pluridisciplinaire HAL, est destinée au dépôt et à la diffusion de documents scientifiques de niveau recherche, publiés ou non, émanant des établissements d'enseignement et de recherche français ou étrangers, des laboratoires publics ou privés. 


\title{
Interplay between bandwidth-controlled and filling-controlled pressure induced Mott insulator to metal transition in the molecular compound [Au(Et-thiazdt $\left.)_{2}\right]$
}

\author{
B. Brière ${ }^{1}$, J. Caillaux ${ }^{1}$, Y. Le Gal ${ }^{2}$, D. Lorcy ${ }^{2}$, S. Lupi ${ }^{3}$, A. Perucchi ${ }^{4}$, \\ M. Zaghrioui ${ }^{1}$, J.C. Soret ${ }^{1}$, R. Sopracase ${ }^{1}$ and V. Ta Phuoc ${ }^{1}$ \\ ${ }^{1}$ GREMAN, CNRS UMR 7347-CEA, Université F. Rabelais, \\ UFR Sciences, Parc de Grandmont, F-37200 Tours, France \\ ${ }^{2}$ Univ Rennes, CNRS, ISCR (Institut des Sciences Chimiques de Rennes) - UMR 6226, F-35000 Rennes, France \\ ${ }^{3}$ CNR-IOM and Dipartimento di Fisica, Università di Roma Sapienza, P.le Aldo Moro 2, I-00185 Roma, Italy and \\ ${ }^{4}$ Elettra - Sincrotrone Trieste S.C.p.A., AREA Science Park, I-34149 Basovizza, Trieste, Italy
}

(Dated: January 24, 2018)

\begin{abstract}
Optical properties of the quasi 2D single-component molecular Mott insulator [Au(Et-thiazdt $)_{2}$ ] (Et-thiazdt $=$ N-ethyl-1,3-thiazoline-2-thione-4,5-dithiolate) have been investigated under pressure at room temperature. At $1.5 \mathrm{GPa},\left[\mathrm{Au}(\text { Et-thiazdt })_{2}\right]$ undergoes an insulator to metal transition (IMT). Optical conductivity spectra exhibit a clear Drude peak at high pressure. In addition, we observed a clear anisotropy of pressure induced modifications of the electronic structure. With increasing pressure, along the molecules stacks, a strong increase of the spectral weight below $1 \mathrm{eV}$ is observed, while in the transverse direction, it remains barely constant with a redistribution from mid-infrared to low energy. Besides the increase of SOMO bandwidth (Singly Occupied Molecular Orbital), calculations show that the SOMO-1 bands cross the Fermi level at the transition. Moreover, we have calculated the optical conductivity as function of pressure to provide a picture of the compound physics under $1 \mathrm{eV}$. Our results indicate that the pressure induced IMT is simultaneously due to a bandwidth and a bandfilling phenomenon that imply both Mott physics and uncorrelated charge carriers.
\end{abstract}

One fascinating phenomenon in strongly correlated fermion systems is the transition from an insulator to a metallic state driven by electronic correlations. An electron in a crystal goes from localized to itinerant (or vice versa) when a control parameter such as doping, pressure or temperature is modified. For a half-filled system, this problem is known as the Mott transition [1]. Understanding the properties of Mott insulators has become of primary importance since the discovery of molecular and high-Tc superconductors $22 \sqrt{5}$ which have in common the fact that from half filled band insulator they become metallic and superconductor with doping (so called "doped Mott insulator"). On this basis, extensive efforts have been devoted to develop new types of molecular metals and superconductors by enhancing the interstack interactions [6]. One solution to raise conductivity and to overstep the charge repulsion issue is to increase the electronic bandwidth $\mathrm{W}$ with respect to the on-site Coulomb repulsion $\mathrm{U}$ which can easily be done by applying pressure [7, 8], especially in organic material due to their soft nature. It has been extensively studied in Mott organic salts like the one-dimensional (1D) tetramethyl-tetrathiafulvalene TMTTF 911 or the two-dimensional (2D) $\kappa$ (Bis(ethylenedithio)tetrathiafulvalene) ${ }_{2} \mathrm{X}$ (BEDTTTF) [12, 13. Moreover, crystals of molecular conductors present numerous advantages for organic electronics compared to traditional inorganic materials: low fabrication cost, high mechanical flexibility, light weight and ease of fabrication [14. For this purpose, the use of neutral organic radicals as building blocks is a promising track to design material with unpaired electrons which serve as charge carriers without any doping process [15].

Among single-component organic conductors based on bis(1,2-dithiolene) ligands [6, 16,21, [Au(Etthiazdt $)_{2}$ (Et-thiazdt $=$ N-ethyl-1,3-thiazoline-2thione-4,5-dithiolate) is the first well characterized molecular metal without TTF dithiolate ligands. It also is the first molecular member of a new class of Resistive Random Access Memory (RRAM) called "Mott Memories". RRam applications are made possible by the resistive switching phenomenon (resistive transition induced by electric pulses). Whereas the resistive switching is usually obtained by electro-chemical effects or charge trapping, it is based on a pure electronic intrinsic effect in inorganic Mott $\mathrm{GaTa}_{4} \mathrm{Se}_{8}$ [22] and in $\left[\mathrm{Au}(\mathrm{Et} \text {-thiazdt })_{2}\right]$ [23. This phenomenon could constitute a generic feature of Mott insulators. Indeed, with a 2D structure made of molecules stacked along b-axis and interstacked along a-axis (see supplemental material [24 part I.) $\left[\mathrm{Au}(\text { Et-thiazdt })_{2}\right]$ is a half-filled electron system with significant electronic correlations that place it close to an IMT controlled by $\mathrm{U} / \mathrm{W}$. Pressure reduces $\mathrm{U} / \mathrm{W}$ sufficiently to induce the IMT at $1.3 \mathrm{GPa}[19$.

In this paper we report a pressure-dependent optical study of $\left[\mathrm{Au}(\mathrm{Et} \text {-thiazdt })_{2}\right]$ single crystals made possible by use of a home-made high-vacuum micro-spectrometer and a synchrotron radiation source (see supp. mat. 24] and reference [25]). In order to understand the mechanisms of the IMT, we performed first-principles Density Functional Theory calculations (DFT). The study highlights the "Mottness" of the compound and describes a new type of Mott transition which involves two types of charge carriers.

In order to elucidate the ambient pressure electronic structure at room temperature, we measured polarized optical conductivity (see Fig,11] [26, 27]. The spectra exhibit an insulating behavior in both $\mathbf{E}|| \mathbf{a}$ and $\mathbf{E} \| \mathbf{b}$ 


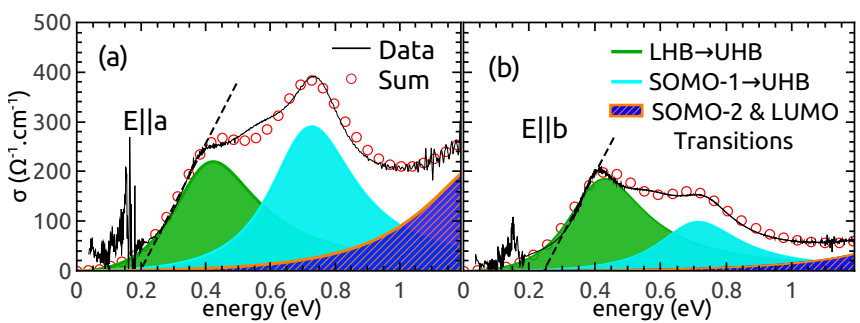

Figure 1. Optical conductivity spectra between $43 \mathrm{meV}$ and $1.2 \mathrm{eV}$ for $\mathbf{E} \| \mathbf{a}$ (a) and $\mathbf{E} \| \mathbf{b}$ (b) at ambient pressure and room temperature. Dotted lines provide the gap. Red circles curves represent the sum of all the contributions.

directions. Although both spectra can be decomposed in the mid infrared (MIR) with at least two absorption bands roughly located at $0.43 \mathrm{eV}$ and $0.74 \mathrm{eV}$, they differ in shape and level. Along a-axis, the amplitude of the $0.74 \mathrm{eV}$ band is higher than the $0.43 \mathrm{eV}$ one. Along b-axis, it is the contrary and the spectrum also exhibits an overall lower absolute level. Despite these differences, the optical gap is roughly the same in both directions $(250 \mathrm{meV})$, in good agreement with transport experiments and previous optical measurements [23] (see supp. mat. 24] part I for the gap extraction procedure).

In order to assign the absorption features to electronic transitions, we performed ab-initio calculations [28 31. Calculation details are provided in the supplemental material [24]. Although DFT does not fully include electronic correlations, it provides valuable information as evidenced in various studies [15, 32. The band structure with "fatband" representation shown in Figure 2 allows to identify the Singly Occupied Molecular Orbital bands (SOMO) (green), the SOMO-1 (cyan), the SOMO-2 (blue) and the Lowest Unoccupied Molecular Orbital bands (LUMO) (orange). Remarkably, two SOMO bands cross the Fermi level $\epsilon_{F}$ driving the state metallic contrary to experiments. This is a typical signature of strong electronic correlations insofar as DFT underestimates on-site interactions. Moreover the SOMO-1 bands remain below $\epsilon_{F}$ and the bandwidth of SOMO bands $\mathrm{W}=0.37 \mathrm{eV}$. Note that the band dispersion along the $\Gamma-Y$ direction is directly related to electron hopping along $\mathbf{b}$-axis. The $\Gamma-\mathrm{X}$ direction is close to the interstack direction a since $\hat{a c}=91.83(5)^{\circ}[19$. Nevertheless, due to the monoclinic symmetry, the optical conductivity tensor is non-diagonal. The non-zero elements of the tensor are provided in Figure 3 The experimental conductivity along the a-axis (Fig.2.a), involves both $\sigma_{x x}$ and $\sigma_{x z}$ components (see supp. mat. 24] part II and reference [33]). $\sigma_{y y}$ is directly compared to the experimental conductivity along $\mathbf{b}$ (Fig.1.b) 1 .

Remarkably, $\sigma_{x x}$ and $\sigma_{x z}$ are dominated by an interband contribution SOMO- $\rightarrow$ SOMO at $0.4 \mathrm{eV}$ (cyan curves Fig 3). Note that a small intraband contribution (green curve) appears in $\sigma_{x x}$ due to the presence of SOMO bands at $\epsilon_{F}$. Moreover, two interband transitions $\mathrm{SOMO}-2 \rightarrow \mathrm{SOMO}$ and $\mathrm{SOMO} \rightarrow \mathrm{LUMO}$ are

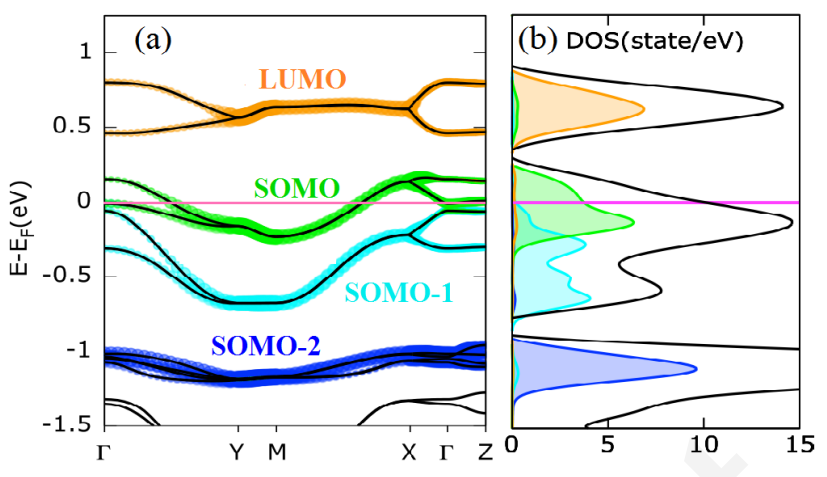

Figure 2. (a): Band structure with 'fatbands' representation calculated at $0 \mathrm{GPa}$, showing the molecular orbitals character. (b): Density Of States (DOS).

predicted around $1 \mathrm{eV}$. SOMO-1 $\rightarrow$ LUMO is predicted around $1.5 \mathrm{eV}$ (above the experimental range). The calculated conductivity for the $\mathbf{E} \| \mathbf{b}$ case $\left(\sigma_{y y}\right)$ is dominated by the SOMO intraband contribution. In addition, $\sigma_{z z}$ indicates an insulating character in agreement with the $2 \mathrm{D}$ character of the system.

It is crucial to notice that the experiment exhibits a first contribution around $0.43 \mathrm{eV}$ (green contribution Fig 1) and a second one around $0.74 \mathrm{eV}$ (cyan contribution) whereas the DFT predicts an intraband (green curve Fig 3 and an interband contribution SOMO$1 \rightarrow \mathrm{SOMO}$ around $0.4 \mathrm{eV}$ (cyan curve). This discrepancy can be understood by taking into account the on-site correlations U. For a large enough U, SOMO bands are split into a filled Lower Hubbard Band (LHB) and an empty Upper Hubbard Band (UHB), resulting in an Mott insulator (Fig, 11.a). By considering the correlations, the DFT intraband SOMO contribution should shift of U, towards higher energies 34. Consequently the contribution at $0.43 \mathrm{eV}$, which has to correspond to the DFT intraband contribution, is assigned to electronic transitions LHB $\rightarrow$ UHB. Moreover, the DFT interband contribution SOMO-1 $\rightarrow$ SOMO should shift of $\mathrm{U} / 2$ towards higher energies in presence of correlations. Therefore, the experimental contribution at $0.74 \mathrm{eV}$ could be assigned to SOMO- $1 \rightarrow$ UHB (empty states of SOMO) excitations. Hence, with an overall good agreement to the experimental data, we deduce $\mathrm{U}=0.43 \mathrm{eV}$. Such a value of $\mathrm{U}$ is commonly found in this class of Mott organic compounds [35, 36. Note that, $\mathrm{U}-\mathrm{W}=0.43-0.37 \approx 0.1 \mathrm{eV}$ which is the same order of the electronic gap. The higher energy excitations $\mathrm{SOMO} \rightarrow \mathrm{LUMO}$ and SOMO-2 $\rightarrow \mathrm{SOMO}$ above the experimental spectral range will not be discussed.

The experimental polarized conductivity spectra presented in Figure 4 from 0 to $5 \mathrm{GPa}$ (at room temperature), exhibit an obvious rising Drude-like response at low frequency above $1.5 \mathrm{GPa}$, a decrease and a closing of the gap between 1 and $1.5 \mathrm{GPa}$ (Fig,4d.). Hence, by applying pressure, $\left[\mathrm{Au}(\text { Et-thiazdt })_{2}\right]$ undergoes an IMT.

Remarkably, the low frequency data extrapolation represented in Figure 4 $\mathrm{c}$ are qualitatively consistent 


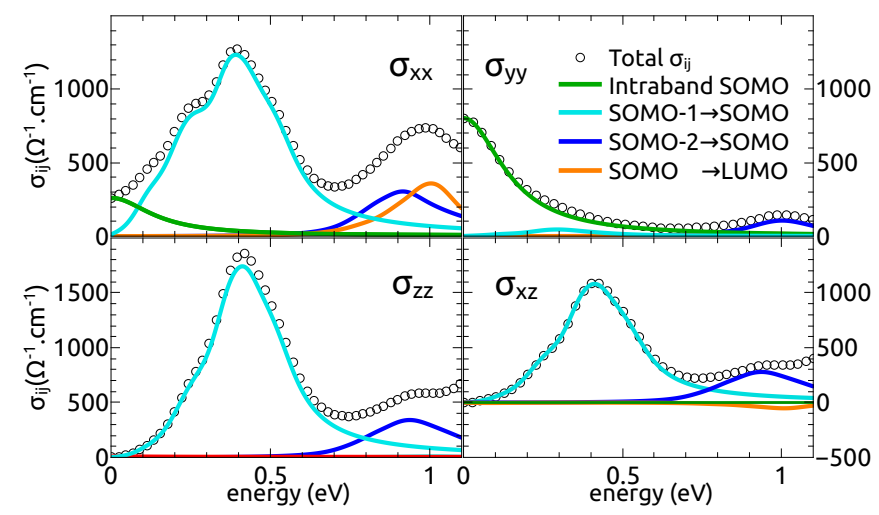

Figure 3. Calculated optical conductivity tensor at ambient pressure.

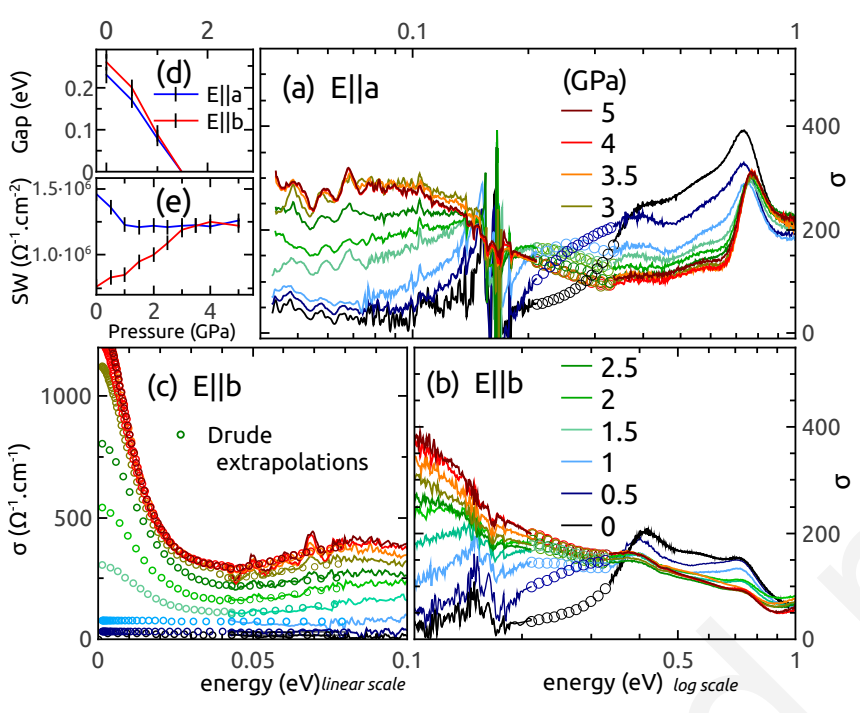

Figure 4. Optical conductivity pressure dependence between $43 \mathrm{meV}$ and $1 \mathrm{eV}$ for $\mathbf{E} \| \mathbf{a}(\mathrm{a})$ and $\mathbf{E} \| \mathbf{b}(\mathrm{b})$ at room temperature. Circles between 210 and $330 \mathrm{meV}$ represent the diamond absorption. (c) shows the low frequency extrapolated behavior of the conductivity along $\mathbf{b}$. This extrapolation was obtained by fitting the low frequency reflectivity $\mathrm{R}_{s d}$ (measured through the diamond) with a Drude model. (d) provides the gap pressure dependence and (e) shows SW calculated for $\omega_{c}=1 \mathrm{eV}$ along both directions.

with room temperature transport measurements realized up to $2.1 \mathrm{GPa}$ by Tenn et al. 19. Indeed, as shown in Figure 5, they both exhibit the same pressure dependence and order of magnitude. Note that this extrapolation was obtained by fitting the low frequency reflectivity $\mathrm{R}_{s d}$ (measured through the diamond) with a Drude model. Also, error bars were extracted by evaluating both the highest and the lowest DC values obtained within the Drude model framework, which reasonably fit the experimental data.

The IMT exhibits several features consistent with the Dynamical Mean Field Theory (DMFT) picture of the Mott transitions [37, 38]: the rise of a Drude peak due to a coherent quasi-particle response and the persistence

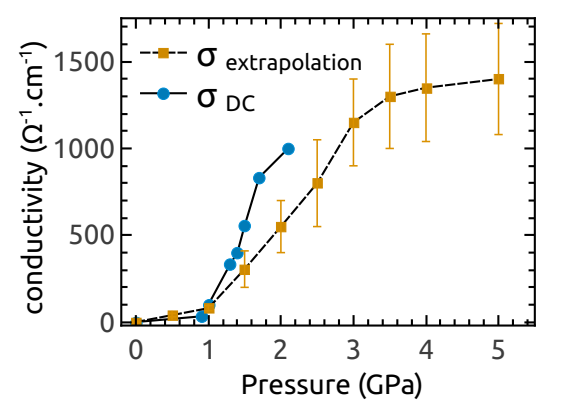

Figure 5. Comparison between room temperature $\sigma_{D C}$ values (up to $2.1 \mathrm{GPa}$ ) obtained by transport measurements by Tenn et al. 19] and by extrapolation of the optical conductivity data at $\omega=0$. They are qualitatively consistent according to both the order of magnitude and the pressure dependence.

of the main MIR absorption band at $0.43 \mathrm{eV}$ assigned to LHB $\rightarrow$ UHB transitions at $0 \mathrm{GPa}$.

In order to rule out possible structural change (from which could originate the IMT), we performed vibrational properties using infrared and Raman spectroscopies. The phonon frequencies as a function of pressure obtained by fitting the infrared and Raman spectra with Drude-Lorentz oscillators and Gaussian-Lorentzian profiles respectively are reported in the supplemental material 24]. Within experimental resolution, apart the usual blue shift of phonon modes (due to the volume lattice shrinking) no phonon anomaly (disappearance, appearance, splitting, kink in the phonon frequency pressure dependence) has been noticed at $1.5 \mathrm{GPa}$ and beyond, in both infrared and Raman spectra. This indicates that no structural phase transition has been detected under pressure in agreement with the Mott transition picture.

Note that the anisotropy still persists at high pressure as it is clearly evidenced by the higher absolute level of the low frequency conductivity along $\mathbf{b}$ than $\mathbf{a}$ and the spectral weight (SW) values (see Fig 4 e). In the b direction, SW increases between 0 and $3 \mathrm{GPa}$ and is constant above $3 \mathrm{GPa}$, whereas along a, it slightly decreases between 0 and $1.5 \mathrm{GPa}$ and is constant above $1.5 \mathrm{GPa}$.

In order to understand the pressure effects on the electronic structure and to disentangle the IMT mechanisms, we performed a full optimization of cell parameters and internal coordinates at different pressures between 0 and 4 GPa within DFT [39], as successfully done for other organic molecular compounds [15, 40].

Pressure effects on band structure are depicted in Figure 6. Note that the lack of dispersion along $\Gamma-\mathrm{Z}$ indicates that the system mainly remains $2 \mathrm{D}$ at high pressure. In addition, the bands shape is almost unchanged between 0 and $4 \mathrm{GPa}$ but the bandwidth increases from $\mathrm{W}=0.37$ to $1.05 \mathrm{eV}$ for the $\mathrm{SOMO}$ and from 0.70 to $1.05 \mathrm{eV}$ for the SOMO-1 bands. As a conse- 


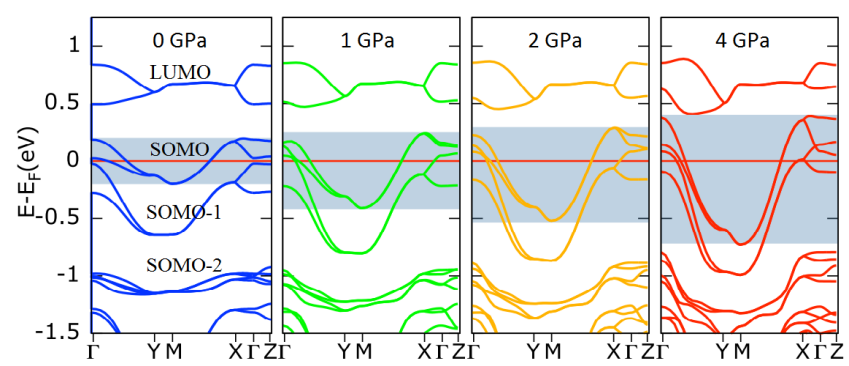

Figure 6. Pressure dependence of band structure calculated by DFT. Grey zones underline the bandwidth of the SOMO bands.

quence, $\mathrm{U} / \mathrm{W}$ goes from 1.16 at $0 \mathrm{GPa}$ to 0.41 at $4 \mathrm{GPa}$, well below the critical value of $\mathrm{U} / \mathrm{W} \approx 1$ for which IMT is theoretically expected to occur [41,42. Consequently, the role of correlations is expected to be weaker when pressure increases and charge carriers are supposed to be less correlated. In addition, as shown in Figure 7. the evolution of the bandwidth $\mathrm{W}$ and the pressure dependence of the damping $\gamma$ of the optical conductivity contribution assigned to LHB $\rightarrow$ UHB transitions are compatible with an usual bandwidth enlargement observed in correlated $2 \mathrm{D}$ organic conductors through the Mott transition [12, 43]. The damping was obtained by fitting the Hubbard mid-infrared absorption band from 0 to $5 \mathrm{GPa}$ with a Drude-Lorentz model.

Remarkably, the increase of dispersion in both $\Gamma$ $\mathrm{X}$ and $\Gamma-Y$ directions raises the energy level of the SOMO-1 bands going at $\Gamma$ from $0^{-}$to $0.10 \mathrm{eV}$ between 0 and $2 \mathrm{GPa}$. As a result, the SOMO- 1 bands cross $\epsilon_{F}$ and become hole filled. In contrast, the SOMO bands (previously assigned to UHB and LHB when correlations are included) go slightly deeper under $\epsilon_{F}$ with pressure, getting electron filled. Hence, part of the IMT is achieved by tuning the electronic filling of the UHB suggesting a pressure induced doping of the Mott insulator. Thus, two mechanisms are driving the IMT: the bandwidth enlargement and the bandfilling. Pressure induces a bandwidth enlargement and an electronic doping of the SOMO bands (UHB) that implies physics related to correlated electrons ('Mottness'). On the contrary, since the SOMO-1 bands are far from half-filling at 0 and $4 \mathrm{GPa}$, the charge carriers arising from hole doping of SOMO-1 bands are expected to be less- or un-correlated.

Comparing the conductivity tensors calculated at $4 \mathrm{GPa}$ (Fig, 8) and at $0 \mathrm{GPa}$ (Fig.3) we notice an enhancement of the low frequency conductivity for both $\sigma_{x x}$ and $\sigma_{y y}$ driving the IMT. Moreover, the SOMO$1 \rightarrow \mathrm{SOMO}$ contribution is shifted of $\approx 0.2 \mathrm{eV}$ towards low frequency and its amplitude is also clearly lower than at ambient pressure. In contrast, for $\sigma_{z z}$, apart from the decrease of the magnitude of the SOMO- $\rightarrow$ SOMO contribution between 0 and $4 \mathrm{GPa}$, there is no significant changes at low frequency. Hence the system remains 2D as previously observed. Remarkably, the Drude contri-

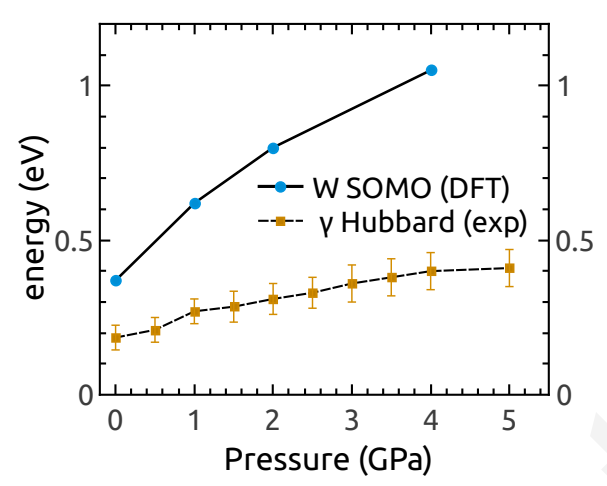

Figure 7. Pressure dependence of the SOMO bandwidth W calculated by DFT. Pressure dependence of the experimental $\mathrm{LHB} \rightarrow \mathrm{UHB}$ contribution damping $\gamma$. They both increase with pressure and they are in agreement with an usual bandwidth enlargement observed in correlated organic 2D conductors through the Mott transition [12, 43].

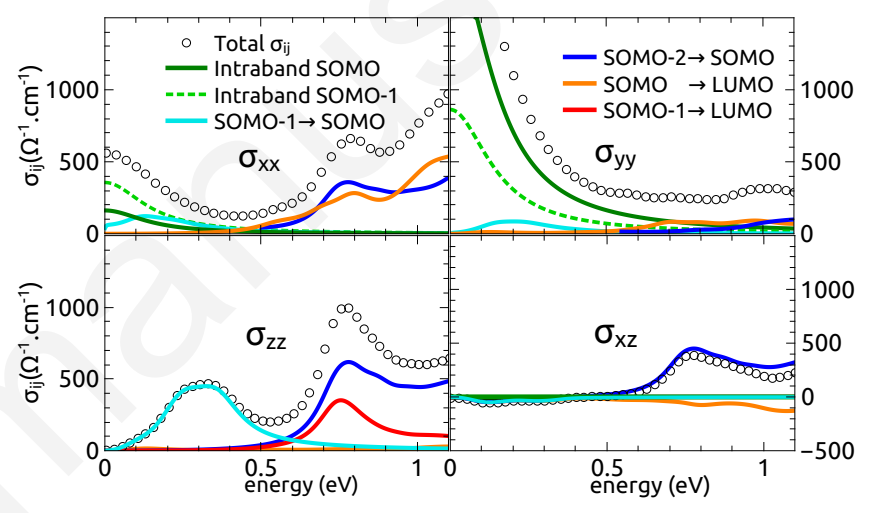

Figure 8. Calculated optical conductivity tensor at 4 GPa.

bution present in $\sigma_{x x}$ and $\sigma_{y y}$ is composed of two different intraband contributions: one due to SOMO bands and an other one produced by the hole filling of the SOMO-1 bands (cf. green curves of Fig, 8). Note that the $\mathrm{SW}$ of the total Drude is lower in $\sigma_{x x}$ than in $\sigma_{y y}$, underlining the remaining anisotropy at high pressure.

In order to get a global picture of the IMT, we both plot in Figure 9 the calculated (deduced from the nondiagonal optical conductivity tensor) and experimental

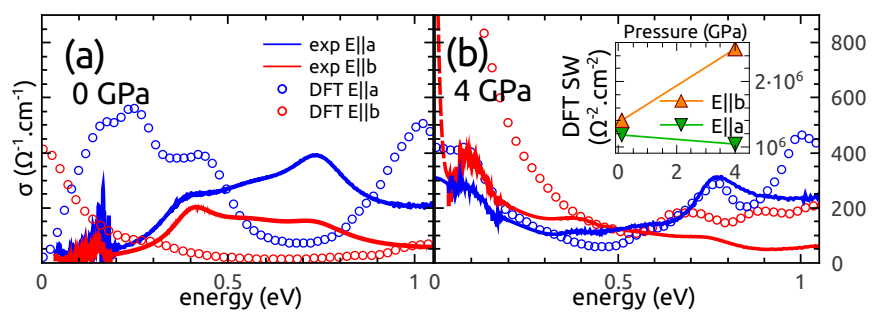

Figure 9. Comparison between calculated and experimental conductivities, for 0 and $4 \mathrm{GPa}$ (a) and (b). Inset : DFT SW calculated at $1 \mathrm{eV}$. 


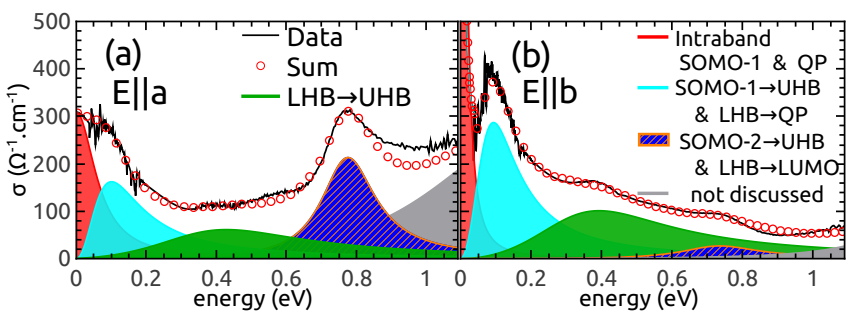

Figure 10. Contributions of optical conductivity spectra for $\mathbf{E} \| \mathbf{a}(\mathrm{a})$ and $\mathbf{E} \| \mathbf{b}(\mathrm{b})$ at $4 \mathrm{GPa}$ and room temperature.

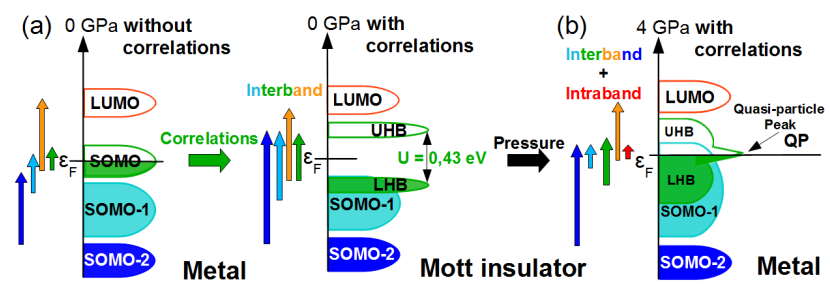

Figure 11. Schematic representation of the electronic structure. (a) Effects of correlations. (b) Effects of pressure. The vertical arrows represent the electronic transitions.

optical conductivity at 0 and $4 \mathrm{GPa}$ along $\mathbf{a}$ and $\mathbf{b}$ axis. Calculations at $4 \mathrm{GPa}$ in both directions are in good agreement with the experiment in many respects. Along the a-axis, the DFT SW (see inset Figure 9.b) decreases slightly from 0 to $4 \mathrm{GPa}$ and along $\mathbf{b}$, it increases between 0 and $4 \mathrm{GPa}$. Moreover, the low frequency DFT conductivity level is consistent with the anisotropy evidenced experimentally since $\sigma_{b}{ }^{D F T}>\sigma_{a}{ }^{D F T}$ at high pressure. Noticeably, the agreement between DFT and experiments is clearly better at high pressure since the role of electronic correlations is expected to be weaker than at ambient pressure.

Naively, in correlated systems, we could consider that the electronically active bands at $\epsilon_{F}$ are ruled/dominated by electronic correlations. However, for $\left[\mathrm{Au}(\mathrm{Et} \text {-thiazdt })_{2}\right]$, other uncorrelated interactions seem to occur. The high pressure experimental conductivity can be fitted under $1 \mathrm{eV}$ with a minimal model consisting of three Lorentz oscillators and one Drude peak (Fig 10). By comparing the experimental and theoretical results (done at $0 \mathrm{GPa}$ ), a schematic view of the electronic structure in the high pressure phase is depicted in Figure 11 b. Note that at $4 \mathrm{GPa}$, DFT reproduces quite well the experimental data except the contribution centered at $0.40 \mathrm{eV}$ (green contribution of Fig 10 which is missing in DFT (Fig 8). Accurately, the two Drude contributions predicted by DFT (dark and dashed green curves Fig 80 are included in the experimental Drude (red contribution of Fig 10). They are assigned to two types of intraband transitions since the SOMO-1 bands and the Quasi-Particle peak (QP) cross $\epsilon_{F}$. Note that QP originates from the bandwidth enlargement of the Hubbard bands as predicted by DMFT [38. The lower frequency interband con- tribution at $\approx 0.2 \mathrm{eV}$ (cyan contribution Fig 10 ) is assigned to both SOMO- $\rightarrow$ SOMO and LHB $\rightarrow$ QP transitions. Note that the LHB $\rightarrow$ QP transitions usually cannot be identified unambiguously in Mott organic compounds because the coherent response is not clearly separated from the LHB $\rightarrow$ UHB excitations [43, 44. At $4 \mathrm{GPa}$ the empty states of the SOMO bands are composed of the UHB and the empty states of the QP peak. They are 'the unoccupied Hubbard states'. Therefore, at $4 \mathrm{GPa}$, SOMO-1 $\rightarrow \mathrm{SOMO}$ transitions are equivalent to SOMO-1 $\rightarrow$ 'unoccupied Hubbard states' transitions. The SOMO-1 $\rightarrow \mathrm{SOMO}$ contribution shifts from $0.74 \mathrm{eV}$ (cyan contribution Fig 1 ) to $\approx 0.2 \mathrm{eV}$ between 0 and $4 \mathrm{GPa}$ because the SOMO-1 bands and UHB are getting closer together with pressure. Hence, the energy required for the transition between SOMO1 bands and 'unoccupied Hubbard states' is reduced. Similarly, as predicted by DFT, the energy required to induce SOMO- $2 \rightarrow \mathrm{SOMO}$ and $\mathrm{SOMO} \rightarrow$ LUMO transitions (blue and orange curves Fig 8 is reduced going from $\approx 1 \mathrm{eV}$ to $\approx 0.75 \mathrm{eV}$ between 0 and $4 \mathrm{GPa}$ due to bandwidth enlargement. Therefore, these contributions are found at $\approx 0.75 \mathrm{eV}$ (blue/orange dashed contribution Fig 10. They are not clearly detected at $0 \mathrm{GPa}$ because they are located beyond $1 \mathrm{eV}$, but with a rough estimation of the correlations effect, we find $1 \mathrm{eV}+\mathrm{U} / 2 \approx 1.2 \mathrm{eV}$ which is consistent with the onset of the contribution at $1.2 \mathrm{eV}$ (blue/orange dashed contribution Fig.11).

Furthermore, the experimental contribution located at $0.4 \mathrm{eV}$ (green contribution Fig 10 and unpredicted by DFT, should correspond to a reminiscence of LHB $\rightarrow$ UHB transitions as predicted by DMFT.

In conclusion, a novel Mott IMT induced by pressure has been evidenced in the organic compound $[\mathrm{Au}(\mathrm{Et}-$ thiazdt $\left.)_{2}\right]$. The originality of the transition lies in the existence of two cooperative mechanisms (bandwidth and bandfilling) that drive the state metallic. On the one hand, SOMO-1 bands are filled with holes underlining the existence of uncorrelated charge carriers. On the other hand, the bandwidth enlargement of Hubbard bands and the filling (doping) of the UHB imply the presence of correlated carriers. Therefore, two types of charge carriers are expected to co-exist in the metallic phase as it appears in other intriguing systems [45 47. Moreover considering the suggested 'doped Mott insulator' character of the system at high pressure, we could expected rich physics at low temperature as encountered for example in cuprates.

The authors wish to thank E. Janod and L. De Medici for fruitful discussions.

[1] P. Limelette, A. Georges, D. Jérome, P. Wzietek, P. Metcalf, and J. M. Honig. Universality and critical behavior at the Mott transition. Science, 302 89, (2003). 
[2] D. Jérome, A. Mazaud, M. Ribault, and K. Bechgaard. Superconductivity in a synthetic organic conductor (TMTSF) $)_{2} \mathrm{PF}_{6}$. J. Phys. Lett. 41, 4, 95-98, (1980).

[3] J. Paglione and R. L. Greene. High-temperature superconductivity in iron-based materials. Nat. Phys. Vol. 6, (2010).

[4] J. L. Tallon, C. Bernhard, H. Shaked, R. L. Hitterman, and J. D. Jorgensen. Generic superconducting phase behavior in high-Tc cuprates: Tc variation with hole concentration in $\mathrm{YBa}_{2} \mathrm{Cu}_{3} \mathrm{O}_{7-\delta}$. Phys. Rev. B. 51, 12911(R), (1995).

[5] H. Oike, K. Miyagawa, H. Taniguchi, and K. Kanoda. Pressure-induced Mott transition in an organic superconductor with a finite doping level. Phys. Rev. Lett. 114, 067002, (2015).

[6] A. Kobayashi, E. Fujiwara, and H. Kobayashi. Singlecomponent molecular metals with extended-TTF dithiolate ligands. Chem. Rev. 104 (11), pp 5243-5264, (2004).

[7] J. W. L. Wong, A. Mailman, K. Lekin, S. M. Winter, W. Yong, J. Zhao, S. V. Garimella, J. S. Tse, R. A. Secco, S. Desgreniers, Y. Ohishi, F. Borondics, and R. T. Oakley. Pressure induced phase transitions and metallization of a neutral radical conductor. J. Am. Chem. Soc. 136, 1070 - 1081, (2014).

[8] A. G. Lebed. The physics of organic superconductors and conductors. (Springer, (2008).

[9] H. Mori. Materials viewpoint of organic superconductors. J. Phys. Soc. Japan, Vol. 75, No. 5, 051003, (2006).

[10] A. Pashkin, M. Dressel, and C. A. Kuntscher. Pressureinduced deconfinement of the charge transport in the quasi-one-dimensional Mott insulator (TMTTF) ${ }_{2} \mathrm{AsF}_{6}$. Phys. Rev. B. 74, 165118, (2006).

[11] M. Tanaka, K. Yamamoto, M. Uruichi, T. Yamamoto, K. Yakushi, S. Kimura, and H. Mori. Infrared and raman study of the charge-ordered state in the vicinity of the superconducting state in the organic conductor $\kappa$ (meso-DMBEDT-TTF ${ }_{2} \mathrm{PF}_{6}$. J. Phys. Soc. Japan Vol. 77, No. 2, 024714, (2008).

[12] M. Dumm, D. Faltermeier, N. Drichko, M. Dressel, C. Mézière, and P. Batail. Bandwidth-controlled Mott transition in $\kappa$-(BEDT-TTF $)_{2} \mathrm{Cu}\left[\mathrm{N}(\mathrm{CN})_{2}\right] \mathrm{Br}_{x} \mathrm{Cl}_{1-x}$ :Optical studies of correlated carriers. Phys. Rev. B. 79, 195106, (2009).

[13] S. Yasin, M. Dumm, B. Salameh, P. Batail, C. Mezière, and M. Dressel. Transport studies at the Mott transition of the two-dimensional organic metal $\kappa$-(BEDTTTF $)_{2} \mathrm{Cu}\left[\mathrm{N}(\mathrm{CN})_{2}\right] \mathrm{Br}_{x} \mathrm{Cl}_{1-x}$. Eur. Phys. J. B. 79: 383, (2011).

[14] N. Koch. Organic electronic devices and their functional interfaces. Chem. Phys.Chem. Vol. 8, Issue 10, (2007).

[15] M. Souto, HB. Cui, M. Peña-Álvarez, V. G. Baonza, H. O. Jeschke, M. Tomic, R. Valentí, D. Blasi, I. Ratera, C. Rovira, and J. Veciana. Pressure-induced conductivity in a neutral nonplanar spin-localized radical. J. Am. Chem. Soc. 138, 11517-11525, (2016).

[16] HB. Cui, H. Kobayashi, S. Ishibashi, M. Sasa, F. Iwase, R. Kato, and A. Kobayashi. A single component molecular superconductor. J. Am. Chem. Soc. 136, 7619, (2014).

[17] O.J. Dautel, M. Fourmigué, E. Canadell, and P. AubanSenzier. Fluorine segregation controls the solid-state organization and electronic properties of $\mathrm{Ni}$ and $\mathrm{Au}$ dithiolene complexes: Stabilization of a conducting singlecomponent gold dithiolene complex. Adv. Funct. Mater. 12, 693-698, (2002).
[18] D. Belo, H. Alves, E. Branco Lopes, M. T. Duarte, V. Gama, R.T. Henriques, M. Almeida, A. PérezBenítez, C. Rovira, and J. Veciana. Gold complexes with dithiothiophene ligands: A metal based on a neutral molecule. Chem. Eur. J. 7, 2, 511-519, (2001).

[19] N. Tenn, N. Bellec, O. Jeannin, L. Piekara-Sady, P. Auban-Senzier, J. Íñiguez, E. Canadell, and D. Lorcy. A single-component molecular metal based on a thiazole dithiolate gold complex. J. Am. Chem. Soc. 131, 1696116967, (2009).

[20] T. Higashino, O. Jeannin, T. Kawamoto, D. Lorcy, T. Mori, and M. Fourmigué. A single-component conductor based on a radical gold dithiolene complex with alkyl-substituted thiophene-2,3-dithiolate ligand. Inorg. Chem. 54, 9908-9913, (2016).

[21] A. Filatre-Furcate, N. Bellec, O. Jeannin, P. AubanSenzier, M. Fourmigué, J. Íñiguez, E. Canadel, B. Brière, V. Ta Phuoc, and D. Lorcy. Single-component conductors: A sturdy electronic structure generated by bulky substituents. Inorg. Chem. 55, 6036-6046, (2016).

[22] L. Cario, C. Vaju, B. Corraze, V. Guiot, and E. Janod. Electric-field-induced resistive switching in a family ofMott insulators: Towards a new class of RRAM memories. Adv. Mat. Vol. 22, Issue 45, (2010).

[23] P. Stoliar, P. Diener, J. Tranchant, B. Corraze, B. Brière, V. Ta-Phuoc, N. Bellec, M. Fourmigué, D. Lorcy, E. Janod, and L. Cario. Resistive switching induced by electric pulses in a single-component molecular mott insulator. J. Phys. Chem. C. 119, 2983-2988, (2015).

[24] See supplemental material for experimental and computa- tional details, data analysis. (2018).

[25] A. Sacchetti, E. Arcangeletti, A. Perucchi, L. Baldassarre, P. Postorino, S. Lupi, N. Ru, I. R. Fisher, and L. Degiorgi. Pressure dependence of the charge-densitywave gap in rare-earth tri-tellurides. Phys. Rev. Lett. 98, 026401, (2007).

[26] A. B. Kuzmenko. Kramers-Kronig constrained variational analysis of optical spectra. Rev. Sci. Instrum. 76, 083108, (2005).

[27] M. Dressel and G. Grüner. Electrodynamics of solids. Cambridge University Press, (2002).

[28] M. Parrinello and A. Rahman. Crystal structure and pair potentials: A molecular-dynamics study. Phys. Rev. Lett. 45, 1196, (1980).

[29] P. Giannozzi et al. Quantum espresso: a modular and open-source software project for quantum simulations of materials. J.Phys.Condens.Matter, 21, 395502, (2009).

[30] Y. Li K. Foyevtsova, H. O. Jeschke, and R. Valentí. Supp. Inf. Analysis of the optical conductivity for $\mathrm{A}_{2} \mathrm{IrO}_{3}(\mathrm{~A}=\mathrm{Na}, \mathrm{Li})$ from first principles. Phys. Rev. B. 91, 161101(R), (2015).

[31] B. Briere, A. Kalinko, I. Yamada, P. Roy, J. B. Brubach, R. Sopracase, M. Zaghrioui, and V. Ta Phuoc. On the energy scale involved in the metal to insulator transition of quadruple perovskite $\mathrm{EuCu}_{3} \mathrm{Fe}_{4} \mathrm{O}_{12}$ : infrared spectroscopy and ab-initio calculations. Sci. Rep. 6:28624, (2016).

[32] A. C. Jacko, H. Feldner, E. Rose, F. Lissner, M. Dressel, R. Valentí, and H. O. Jeschke. Electronic properties of fabre charge-transfer salts under various temperature and pressure conditions. Phys. Rev. B. 87, 155139, (2013).

[33] V. Ta Phuoc, C. Sellier, E. Janod, and C. Marin. Polarized reflectivity of $\beta-\mathrm{Sr}_{0.17} \mathrm{~V}_{2} \mathrm{O}_{5}$. Phys. Rev. B. 77, 075123, (2008).

[34] M. J. Rozenberg, G. Kotliar, H. Kajueter, G. A. 
Thomas, D. H. Rapkine, J. M. Honig, and P. Metcalf. Optical conductivity in Mott-Hubbard systems. Phys. Rev. Lett. 75, 105, (1995).

[35] K. Sedlmeier, S. Elsässer, D. Neubauer, R. Beyer, D. Wu, T. Ivek, S. Tomić, J. A. Schlueter, and M. Dressel. Absence of charge order in the dimerized $\kappa$ phaseBEDT-TTF salts. Phys. Rev. B. 86, 245103, (2012).

[36] S. Yasuzuka and K. Murata. Recent progress in highpressure studies organic conductors. Sci. Technol. Adv. Mater. 10, 024307, (2009).

[37] A. Georges, G. Kotliar, W. Krauth, and M. J. Rozenberg. Dynamical mean-field theory of strongly correlated fermion systems and the limit of infinite dimensions. Rev. Mod. Phys. 68, 13, (1996).

[38] G. Kotliar, S. Y. Savrasov, K. Haule, V. S. Oudovenko, O. Parcollet, and C. A. Marianetti. Electronic structure calculations with dynamical mean-field theory: A spectral Density Functional approach. Rev. Mod. Phys. 78, 865, (2006).

[39] T. H. Fischer and J. Almlof. General methods for geometry and wave function optimization. J. Phys. Chem. 96, 9768, (1992).

[40] X. Juan, W.-H. Zhu, and H.-M. Xiao. DFT studies on the four polymorphs of crystalline CL-20 and the influences of hydrostatic pressure on E-CL-20 crystal. J. Phys. Chem. B. 111, 2090-2097, (2007).

[41] E. Janod, J. Tranchant, B. Corraze, M. Querré, P. Stoliar, M. Rozenberg, T. Cren, D. Roditchev, V. Ta
Phuoc, M-P. Besland, and L. Cario. Resistive switching in mott insulators and correlated systems. Adv. Funct. Mater. vol. 25, 40, 6287-6305, (2015).

[42] S. Karmakar and P. S. Malavi. Novel P-T phase diagram of the multiorbital Mott insulator $\mathrm{Sr}_{2} \mathrm{VO}_{4}$. Phys. Rev. Lett. 114, 166402, (2015).

[43] J. Ferber, K. Foyevtsova, H. Jeschke, and R. Valentí. Unveiling the microscopic nature of correlated organic conductors: The case of $\kappa-(\mathrm{ET})_{2} \mathrm{Cu}\left[\mathrm{N}(\mathrm{CN})_{2} \mathrm{Br}_{x} \mathrm{Cl}_{1-x}\right.$. Phys. Rev. B. 89, 205106, (2014).

[44] M. Dressel, D. Faltermeier, M. Dumm, N. Drichko, B. Petrov, V. Semkin, R. Vlasova, C. Méziere, and P. Batail. Disentangling the conductivity spectra of two-dimensional organic conductors. Physica B. 404, 541-544, (2009).

[45] M. Le Tacon, C. Bernhard, H. Shaked, R. L. Hitterman, and J. D. Jorgensen. Two energy scales and two distinct quasiparticle dynamics in the superconducting state of underdoped cuprates. Nat. Phys. 2, 537 - 543, (2006).

[46] D. Wu, N. Barišić, P. Kallina, A. Faridian, B. Gorshunov, N. Drichko, L. J. Li, X. Lin, G. H. Cao, Z. A. $\mathrm{Xu}$, N. L. Wang, and M. Dressel. Optical investigations of the normal and superconducting states reveal two electronic subsystems in iron pnictides. Phys. Rev. B. 81, 100512, (2010).

[47] L. de Medici, G. Giovannetti, and M. Capone. Selective Mott physics as a key to iron superconductors. Phys. Rev. Lett. 112, 177001, (2014). 